\title{
THE PROJECTIVE DIFFERENTIAL GEOMETRY OF SYSTEMS OF LINEAR HOMOGENEOUS DIFFERENTIAL EQUATIONS OF THE FIRST ORDER*
}

\author{
BY \\ ERNEST P. LANE

\section{INTRODUCTION}

The tedious calculations that occasionally occur in studying projective differential geometry by means of the invariants and covariants of a completely integrable system of linear homogeneous differential equations are admittedly an unpleasant feature of this method. But it seems that labor can sometimes be avoided by reducing the system of equations to a system of the first order. Such a system proves to be especially suitable for the study of a configuration composed of a set of varieties generated by linear spaces, with the generators in correspondence.

The present paper is concerned for the most part with the projective differential geometry that can be studied by means of a system of $n+1$ linear homogeneous differential equations of the first order in $n+1$ dependent variables and one independent variable. The transformation of dependent variables that is used is determined by the configuration to be studied, which in any case consists of a set of varieties each of which is generated by $\infty^{1}$ linear spaces, with the generators in correspondence. The precise number of possible configurations of this type in a space of a given number of dimensions is determined, but by imposing the condition that some of the varieties shall be covariant to the rest of them the range of applicability of the method can be widely extended. Certain curves called intersector curves on a variety are defined, and the locus of their tangents is investigated.

A section is devoted to geometry in a space $S_{5}$ of five dimensions. The intersection of a configuration with a hyperquadric in $S_{5}$ yields a geometric interpretation in ordinary ruled space. Another section is taken up with a pair of ruled surfaces in ordinary space, with their generators in correspondence. A complete system of invariants is furnished therefor, and a new canonical form of the differential equations is established.

Finally a system of $k(n+1)$ linear homogeneous partial differential equations of the first order in $n+1$ dependent variables and $k$ independent var-

\footnotetext{
* Presented to the Society, December 29, 1927; received by the editors in December,1927.
} 
iables is considered, and the foundations are laid for the projective differential geometry of a configuration composed of a set of varieties each of which is generated by $\infty^{k}$ linear spaces, with the generators in correspondence.

\section{ORDINARY DIFFERENTIAL EQUATIONS}

In a linear space $S_{n}$ of $n$ dimensions let us consider $n+1$ line arly independent points $P_{\boldsymbol{i}}$ with projective homogeneous coördinates

$$
x_{i}^{(1)}, \cdots, x_{i}^{(n+1)} \quad(i=1, \cdots, n+1),
$$

which are single-valued analytic functions of one independent variable $t$. As $t$ varies each point $P_{i}$ describes a curve $C_{i}$, and we thus obtain $n+1$ curves in $S_{n}$ with their points in correspondence, corresponding points being those that correspond to the same value of the parameter $t$.

The square matrix of the coördinates $x_{i}{ }^{(j)}$ of the points $P_{i}$ is of order and rank $n+1$. Therefore it is possible to determine the coefficients of a system of equations of the form

$$
x_{i}^{\prime}=\sum_{j=1}^{n+1} a_{i j} x_{j} \quad(i=1, \cdots, n+1),
$$

so that $\left(x_{1}{ }^{(k)}, \cdots, x_{n+1}^{(k)}\right) \cdot(k=1, \cdots, n+1)$ will be $n+1$ sets of solutions. For example we may substitute each of these sets in turn in the first equation of system $\left(E_{n}\right)$ and then solve the resulting $n+1$ equations for the coefficients of the first of equations $\left(E_{n}\right)$. Similarly, the coefficients of each of the other $n$ equations can be determined.

The transformation of dependent variables

$$
\left(T_{n \cdot 1}\right) \quad x_{i}=\lambda_{i} \bar{x}_{i} \quad(i=1, \cdots, n+1),
$$

and the transformation of independent variable

$$
u=u(t), \quad u^{\prime} \neq 0,
$$

leave each of the curves $C_{i}$ invariant. Therefore the projective differential geometry of a set of $n+1$ curves in $S_{n}$ with their points in correspondence can be studied by means of the invariants and covariants of system $\left(E_{n}\right)$ under the total transformation $\left(T_{n \cdot 1} U\right)$. Various configurations covariant to such curves can be defined at once. For example, $n$ of the points $P_{i}$ corresponding to a value of $t$ determine a hyperplane $S_{n-1}$, and as $t$ varies this hyperplane osculates a curve. In this way $n+1$ new curves with their points in correspondence with those of the original curves are obtained. And for each $k$ $(k=1, \cdots, n-1)$ there are $C_{n+1, k+1}$ covariant varieties $V_{k+1}$ each of which is generated by $\infty^{1}$ linear spaces $S_{k}$ determined by $k+1$ of the points $P_{i}$, and the 
generators of all of these are in correspondence with the points $P_{i}$ of the curves $C_{i}$.

With $a$ different choice of the transformation of dependent variables, the projective differential geometry of a different configuration can be studied by means of the corresponding invariants and covariants of system $\left(E_{n}\right)$. The situation will be made clear by illustrations. If $n=2$, besides the geometry of a triple of plane curves* with their points in correspondence, we can also study by means of $\left(E_{2}\right)$ the geometry of a pair of plane curves with the tangents of one corresponding to the points of the other if we use the transformation of dependent variables

$$
x_{i}=\sum_{j=1}^{2} \lambda_{i j} \bar{x}_{j} \quad(i=1,2), \quad x_{3}=\lambda_{3} \bar{x}_{3} .
$$

Obviously the points of these two curves are in correspondence, and the lines joining corresponding points envelop a new covariant curve, while the point of intersection of the tangents at corresponding points generates another, and so on.

If $n=3$, there are three possibilities besides a quadruple of space curves with their points in correspondence. First of all, if we use the transformation

$$
x_{i}=\sum_{j=1}^{2} \lambda_{i j} \bar{x}_{j}, x_{k}=\lambda_{k} \bar{x}_{k} \quad(i=1,2 ; k=3,4),
$$

we obtain the geometry of a ruled surface and a pair of curves with the generators of the surface and the points of the curves in correspondence. The lines joining corresponding points of the curves generate a covariant ruled surface. In the second place, the transformation

$$
x_{i}=\sum_{j=1}^{2} \lambda_{i j} \bar{x}_{j}, \quad x_{k}=\sum_{j=3}^{4} \lambda_{k j} \bar{x}_{i} \quad(i=1,2 ; k=3,4)
$$

yields the geometry of a pair of ruled surfaces with generators in correspondence which we have studied elsewhere, $\dagger$ and concerning which we shall have more to say in $\$ 4$. Finally, the transformation

$$
x_{i}=\sum_{j=1}^{3} \lambda_{i j} \bar{x}_{j}(i=1,2,3), x_{4}=\lambda_{4} \bar{x}_{4}
$$

gives a developable surface and a curve with the tangent planes of the one and the points of the other in correspondence. The points of the edge of regression

* Jerbert, Abstract, Bulletin of the American Mathematical Society, vol. 33 (1927), p. 516.

$\dagger$ Lane, Ruled surfaces with generators in one-to-one correspondence, these Transactions, vol. 25 (1923), p. 281. 
of the developable and those of the other curve are in correspondence, and the ruled surface of lines joining corresponding points is a covariant ruled surface, as is also the ruled surface of lines of intersection of the osculating planes of the two curves at corresponding points.

In general, the number of configurations whose projective differential geometry can be studied in $S_{n}$ by means of the invariants and covariants of system $\left(E_{n}\right)$ under linear transformations of the dependent variables and the transformation $(U)$ of the independent variable is $n\left(n^{2}+20\right) / 24$ if $n$ is even and $n \geqq 2$; and the number is $n\left(n^{2}+23\right) / 24$ if $n$ is odd. These formulas can be obtained by observing that the number under consideration is the number of ways in which it is possible to choose at least two transformations of the form

$$
\begin{array}{r}
x_{i}=\sum_{i=1}^{a} \lambda_{i j} \bar{x}_{j}(i=1, \cdots, a) ; x_{k}=\sum_{j=a+1}^{b} \lambda_{k j} \bar{x}_{i} \quad(k=a+1, \cdots, b) ; \\
\cdots ; x_{l}=\sum_{j=0+1}^{h} \lambda_{l i} \bar{x}_{i} \quad(l=g+1, \cdots, h),
\end{array}
$$

where $a, b, \cdots, g, h$ are positive integers such that

$$
a \geqq b-a \geqq \cdots \geqq g-f \geqq h-g, \quad a<b<\cdots<g<h=n+1 .
$$

In particular, if $n=4$, the number of configurations is 6 , and if $n=5$, the number is 10 .

Let us consider any one of the possible configurations in $S_{n}$, and any one of the varieties $V_{k+1}$ in this configuration which is the locus of an $S_{k}$ with $1 \leqq k \leqq n-2$. A curve on such a variety will be called an intersector curve with respect to the remaining varieties in the configuration in case the tangent to the curve at the point where it crosses a generator $S_{k}$ intersects the linear space $S_{n-k-1}$ determined by the generators of the other varieties in the configuration that correspond to $S_{k}$. In order to obtain the differential equations of these curves let us observe that any curve on the $V_{k+1}$ can be regarded as the locus of a point $\phi$, where

$$
\phi=x_{1}+\sum_{j=1}^{k+1} \lambda_{j} x_{j}
$$

The point $\phi^{\prime}+h \phi$ is any point on the tangent of this curve at the point $\phi$, and $\phi^{\prime}+h \phi$ can be expressed as a linear combination of $x_{1}, \cdots, x_{n+1}$. If the curve is an intersector curve, then the coefficients of $x_{1}, \cdots, x_{k+1}$ must vanish. Thus we obtain $k+1$ equations, and elimination of $h$ from them gives the differential equations of the intersector curves on $V_{k+1}$ 


$$
\lambda_{p}^{\prime}+\dot{a}_{1 p}-a_{11} \lambda_{p}+\sum_{j=2}^{k+1} \lambda_{j}\left(a_{j p}-\lambda_{p} a_{j 1}\right)=0 \quad(p=2, \cdots, k+1) .
$$

There are $\infty^{k}$ of these curves, one of them passing through each point of a generator $S_{k}$.

Tangents of intersector curves may be called intersector tangents. The locus of the intersector tangents at points of a fixed generator $S_{k}$ is a variety $V_{k+1}$, whose equations can be found in the following way. In the expression for $\phi^{\prime}+h \phi$ as a linear combination of $x_{1}, \cdots, x_{n+1}$ let us replace $\lambda_{p}^{\prime}$ by the expression given therefor by equation (3). Then let us denote the coefficients of $x_{1}, \cdots, x_{n+1}$ by $y_{1}, \cdots, y_{n+1}$ respectively, so that $y_{1}, \cdots, y_{n+1}$ are the local coördinates of the point $\phi^{\prime}+h \phi$ on an intersector tangent referred to the pyramid whose vertices are the points $x_{1}, \cdots, x_{n+1}$. Thus we find the parametric equations of the locus of the intersector tangents at points of a fixed generator,

$$
\begin{aligned}
& y_{1}=a_{11}+\sum_{j=2}^{k+1} \lambda_{j} a_{j 1}+h, y_{p}=\lambda_{p} y_{1} \quad(p=2, \cdots, k+1), \\
& y_{q}=a_{1 q}+\sum_{j=2}^{k+1} \lambda_{j} a_{j q} \quad(q=k+2, \cdots, n+1) .
\end{aligned}
$$

If $2 k>n-2$, homogeneous elimination of $\lambda_{2}, \cdots, \lambda_{k+1}$ and $h$ from these equations gives $n-k-1$ quadratic equations of the locus of the intersector tangents,

$$
\sum_{j=1}^{k+1} y_{j}\left(a_{j q} y_{n+1}-y_{q} a_{j, n+1}\right)=0 \quad(q=k+2, \cdots, n) .
$$

The locus is therefore $a V_{k+1}$ of order $2(n-k-1)$. If, however, $2 k \leqq n-2$, homogeneous elimination gives $n-1-2 k$ linear equations and $k$ quadratic equations. In this case the locus is a $V_{k+1}$ of order $2 k$.

\section{SPACE OF FIVE DIMENSIONS}

The case $n=5$ offers especial interest because the intersection of a configuration with a hyperquadric in $S_{5}$ can be interpreted as a configuration of line geometry in ordinary ruled space. And of the ten possible configurations in $S_{5}$ there are three which, because of their symmetrical properties, seem to be most interesting.

Denoting the equations $\left(E_{n}\right)$ when $n=5$ by $\left(E_{5}\right)$, let us consider in connection therewith the transformation

$$
\begin{aligned}
x_{i}=\sum_{j=1}^{2} \lambda_{i j} \bar{x}_{i}, \quad x_{k}=\sum_{j=3}^{4} \lambda_{k j} \bar{x}_{i}, \quad x_{l}=\sum_{j=5}^{6} \lambda_{l j} \bar{x}_{i} \\
(i=1,2 ; \quad k=3,4 ; \quad l=5,6) .
\end{aligned}
$$


The configuration to be studied is a triple of ruled surfaces with their generators in correspondence. One of these ruled surfaces $R_{12}$ is the locus of the line $l_{12}$ joining the points $P_{1}, P_{2}$; and similar notations will be used for the others.

Considering a point $x_{1}+\lambda x_{2}$ on $l_{12}$ we find that the differential equation of the intersector curves on $R_{12}$ with respect to $R_{34}$ and $R_{56}$ is

$$
\lambda^{\prime}+a_{12}-\left(a_{11}-a_{22}\right) \lambda-a_{21} \lambda^{2}=0 \text {. }
$$

The harmonic property of four particular solutions of an equation of Riccati can be given a new geometric interpretation here. And if $\lambda$ is the general solution of (6) the intersector tangents of $R_{12}$ at points of $l_{12}$ intersect the $S_{3}$ determined by the corresponding generators $l_{34}, l_{56}$ in a straight line which is the locus of the point

$$
\sum_{j=3}^{6}\left(a_{1 j}+\lambda a_{2 j}\right) x_{j}
$$

as the constant of integration takes all possible values. As $t$ varies, this line generates a ruled surface on the $V_{4}$ generated by the $S_{3}$.

The osculating plane of a curve on $R_{12}$ generated by a point $x_{1}+\lambda x_{2} c_{\mu}$ $l_{12}$ ordinarily meets the $S_{3}$ of the corresponding generators $l_{34}, l_{56}$ in a point whose coördinates can easily be found. But if we impose on the curve the condition that the intersection of its osculating plane and the $S_{3}$ shall be a straight line, then there are only two curves of this type on $R_{12}$, and for them $\lambda$ is a root of the equation

$$
\sum_{j=3}^{6}\left[a_{2 j} a_{j 1} \lambda^{2}+\left(a_{1 j} a_{j 1}-a_{2 j} a_{j 2}\right) \lambda-a_{1 j} a_{j 2}\right]=0 .
$$

The effect of $\left(T_{5.4}\right)$ on system $\left(E_{5}\right)$ is to produce another system of the same form. If we choose $\left(\lambda_{11}, \lambda_{21}\right)$ and $\left(\lambda_{12}, \lambda_{22}\right)$ as two pairs of solutions of the differential equations

$$
\phi^{\prime}=a_{11} \phi+a_{12} \psi, \quad \psi^{\prime}=a_{21} \phi+a_{22} \psi,
$$

we find that $\bar{a}_{i j}=0(i, j=1,2)$ : Similarly we can make $\bar{a}_{i j}=0(i, j=3,4)$, and $\bar{a}_{i j}=0(i, j=5,6)$. Thus we obtain a canonical form of system $\left(E_{5}\right)$ for which the directrix curves on each surface are intersector curves with respect to the other two, and for which, moreover, the derivative point of a point $x_{i}$ on one surface lies in the $S_{3}$ determined by the generators of the other two surfaces that correspond to the generator through $x_{i}$.

Another canonical form of $\left(E_{5}\right)$ can be obtained in the following way. Let us define the symbol $(x y)$ by the equation

$$
(x y)=x^{(1)} y^{(8)}+x^{(2)} y^{(5)}+x^{(3)} y^{(4)}+x^{(4)} y^{(8)}+x^{(5)} y^{(2)}+x^{(6)} y^{(1)} \text {. }
$$


Then $(x x)=0$ is the equation of a hyperquadric $V_{4}^{2}$ in $S_{5}$, and a point $\lambda x_{1}+\mu x_{2}$ on $l_{12}$ lies on $V_{4}^{2}$ in case $\lambda: \mu$ satisfies the equation

$$
\left(x_{1} x_{1}\right) \lambda^{2}+2\left(x_{1} x_{2}\right) \lambda \mu+\left(x_{2} x_{2}\right) \mu^{2}=0 .
$$

Assuming that the roots of this equation are distinct, so that $l_{12}$ is not tangent to $V_{4}^{2}$, let us choose one root for $\lambda_{11}: \lambda_{12}$ and the other for $\lambda_{21}: \lambda_{22}$. Then the transformation $\left(T_{5.4}\right)$ makes the new directrix curves $C_{1}, C_{2}$ of $R_{12}$ lie on $V_{4}^{2}$. In this way we can obtain a canonical form of $\left(E_{5}\right)$ for which all six directrix curves lie on $V_{4}^{2}$.

The usefulness of the second canonical form is due to the well known fact that point geometry on $V_{4}^{2}$ in $S_{5}$ is isomorphic with line geometry in ordinary ruled space $R_{3}$. To the curves $C_{1}, C_{2}$ on $V_{4}^{2}$ correspond two ruled surfaces in $R_{3}$. The geometry of the intersection of $V_{4}^{2}$ and a triple of ruled surfaces in $S_{5}$ with their generators in correspondence is equivalent to the geometry of a triple of pairs of ruled surfaces in $R_{3}$ with their generators in correspondence. The assumption that the points $P_{i}$ are linearly independent implies that six corresponding generators in $R_{3}$ do not belong to a linear complex.

A triple of ruled surfaces* in $R_{3}$ with their generators in correspondence is equivalent to a triple of curves on $V_{4}^{2}$ in $S_{5}$ with their points in correspondence. If the ruled surfaces of the triple are given by their parametric equations in line coördinates

$$
x_{i}^{(j)}=x_{i}^{(j)}(t)(i=1,2,3 ; j=1, \cdots, 6),\left(x_{i} x_{i}\right)=0,
$$

and if no three corresponding generators with the generators consecutive to each of them belong to the same linear complex, then on $V_{4}^{2}$ we have three curves whose tangents at corresponding points do not lie in an $S_{4}$. Therefore, the coördinates $\left(x_{1}^{(j)}, x_{2}{ }^{(j)}, x_{3}^{(j)}\right)(j=1, \cdots, 6)$ form six sets of solutions of a system of differential equations of the form

$$
x_{i}^{\prime \prime}=\sum_{j=1}^{3}\left(p_{i j} x_{j}^{\prime}+q_{i j} x_{j}\right) \quad(i=1,2,3),
$$

and the geometry of the triple of ruled surfaces can be studied by means of the invariants and covariants of these equations under the group of transformations

$$
x_{i}=\lambda_{i} \bar{x}_{i} \quad(i=1,2,3), \quad u=u(t) .
$$

Equations (12) can be converted into a system of the form $\left(E_{5}\right)$ by placing $x_{4}=x_{1}^{\prime}, x_{5}=x_{2}^{\prime}, x_{6}=x_{3}^{\prime}$. The appropriate group of transformations is readily determined.

\footnotetext{
- Carpenter, Triads of ruled surfaces, these Transactions, vol. 29 (1927), p. 254.
} 
Let us now consider system $\left(E_{5}\right)$ and the transformation

$$
x_{i}=\sum_{j=1}^{3} \lambda_{i j} \bar{x}_{i}, \quad x_{k}=\sum_{j=4}^{6} \lambda_{k j} \bar{x}_{i} \quad(i=1,2,3 ; k=4,5,6) .
$$

The configuration in this case is a pair of varieties $V_{3}$, each of which is generated by $\infty^{1}$ planes, with the generators in correspondence. We shall denote these varieties by $V_{3}^{\prime}$ and $V_{3}^{\prime \prime}$, the first being generated by the plane $S_{2}^{\prime}$ determined by the points $P_{1}, P_{2}, P_{3}$, and the second by $S_{2}^{\prime \prime}$ determined by $P_{4}$, $P_{5}, P_{6}$.

By an easy extension of the method used in connection with equations (8) it is possible to obtain a canonical form of system $\left(E_{5}\right)$ for which $\bar{a}_{i j}=\bar{a}_{p q}=0$ $(i, j=1,2,3 ; p, q=4,5,6)$. The directrix curves on each variety are then intersector curves with respect to the other, and the derivative point of a point $x_{i}$ on one variety lies in the corresponding generating plane of the other.

Another canonical form of system $\left(E_{5}\right)$ can be obtained by demanding that the directrix curves of $V_{3}^{\prime}$ and $V_{3}^{\prime \prime}$ lie on the hyperquadric $V_{4}^{2}$. A point $\lambda_{1} x_{1}+\lambda_{2} x_{2}+\lambda_{3} x_{3}$ on $S_{2}^{\prime}$ lies on the conic of intersection of $S_{2}^{2}$ and $V_{4}^{2}$ in case $\lambda_{1}, \lambda_{2}, \lambda_{3}$ satisfy the equation

$$
\sum_{i, j=1}^{3}\left(x_{i} x_{j}\right) \lambda_{i} \lambda_{j}=0
$$

Therefore three sets of values of $\lambda_{1}, \lambda_{2}, \lambda_{3}$ which satisfy this equation furnish three directrix curves of $V_{3}^{\prime}$ on $V_{4}^{2}$. Similarly, three directrix curves for $V_{3}^{\prime \prime}$ can be found on $V_{4}^{2}$.

To the conic of intersection of $S_{2}^{\prime}$ and $V_{4}^{2}$ corresponds in $R_{3}$ a regulus composed of one family of generators of a quadric surface. As $t$ varies, the conic generates a surface on $V_{4}^{2}$, and the regulus generates a congruence. The geometry of the intersection of the hyperquadric and the two varieties $V_{3}^{\prime}, V_{3}^{\prime \prime}$ is equivalent to the geometry of two congruences each of which is the locus of $\infty^{1}$ reguli in $R_{3}$, with generating reguli in correspondence. Ordinarily two consecutive reguli of such a congruence do not intersect. But the flecnode congruence of a ruled surface is an example of such a congruence in which two consecutive reguli do intersect in a generator of the ruled surface counted twice. And the congruence of asymptotic tangents of a ruled surface is an example of such a congruence in which two consecutive reguli intersect in two flecnode tangents of the ruled surface.

If we consider finally system $\left(E_{5}\right)$ and the transformation

$$
x_{i}=\sum_{j=1}^{4} \lambda_{i j} \bar{x}_{i}, \quad x_{k}=\sum_{j=5}^{6} \lambda_{k j} \bar{x}_{i} \quad(i=1, \cdots, 4 ; k=5,6),
$$


the configuration is $a V_{4}$, which is the locus of $\infty^{1}$ linear spaces $S_{3}$, and a ruled surface $R_{56}$, with generators in correspondence.

A canonical form of system $\left(E_{5}\right)$ can be obtained for which $\bar{a}_{i j}=\bar{a}_{p q}=0$ $(i, j=1, \cdots, 4 ; p, q=5,6)$. This canonical form is related to the intersector curves on $V_{4}$ and $R_{56}$, as in previous cases.

The intersection of this configuration with $V_{4}^{2}$ is a variety $V_{3}$ generated by $\infty^{1}$ quadrics $V_{2}^{2}$, and two curves which are the loci of points in correspondence with the quadrics. The geometry of this configuration on the hyperquadric is equivalent in $R_{3}$ to the geometry of a complex generated by $\infty^{1}$ linear congruences, and a pair of ruled surfaces whose generators are in correspondence with the congruences. Two consecutive congruences of the complex ordinarily intersect in two lines which generate two ruled surfaces in the complex. Moreover, the axes of a congruence of the complex also generate two ruled surfaces, and these are such that their two common tangents at points of corresponding generators are the aforesaid lines of intersection of two consecutive congruences.

An example of a complex of the type under consideration is the complex generated by the osculating linear congruences of a ruled surface. Such a congruence is determined by four consecutive generators of the ruled surface, and consists of the lines intersecting two flecnode tangents. Two consecutive congruences intersect in an osculating regulus determined by three consecutive generators of the ruled surface. Three consecutive congruences intersect in a generator of the surface.

A second example is the complex of tangents of a ruled surface. This consists of $\infty^{1}$ special linear congruences of tangents at points of a generator. Two consecutive congruences of this kind intersect in a regulus of asymptotic tangents, and three consecutive congruences intersect in two flecnode tangents at points of a generator of the ruled surface.

\section{PAIRS OF RULED SURFACES IN ORDINARY SPACE}

In the paper referred to in $\$ 2$ we studied a pair of ruled surfaces in ordinary space with their generators in correspondence, using a transformation of the form $\left(T_{3 \cdot 3}\right)$, and using the following differential equations:

$\left.E_{3}\right)$

$$
\begin{aligned}
& y^{\prime}=c_{11} y+c_{12} z+a_{11} \rho+a_{12} \sigma \\
& z^{\prime}=c_{21} y+c_{22} z+a_{21} \rho+a_{22} \sigma \\
& \rho^{\prime}=b_{11} y+b_{12} z+d_{11} \rho+d_{12} \sigma \\
& \sigma^{\prime}=b_{21} y+b_{22} z+d_{21} \rho+d_{22} \sigma .
\end{aligned}
$$

For every configuration studied by our method the problem of computing 
a complete system of invariants arises. The method ordinarily used is that of infinitesimal transformations according to Lie's theory of continuous groups. This method applied to system $\left(E_{3}\right)$ leads to the following results. A complete system of seminvariants, i.e., invariants under $\left(T_{3.8}\right)$, of a pair of ruled surfaces with generators in correspondence consists of the eight seminvariants $I_{2}, I_{4}, J_{4}, J_{8}, K_{3}, K_{5}, K_{7}, L_{6}$ defined by the following formulas:

$$
\begin{aligned}
I_{2} & =\sum a_{i j} b_{i j}, J_{4}=\sum A_{i j} B_{i j}, K_{3}=\sum A_{i j} b_{i j}, L_{6} \equiv \sum \mathfrak{A}_{i j} \mathfrak{Z}_{i j} \\
I_{4} & =(i, j=1,2), \\
J_{8} & =\left(a_{11} a_{22}-a_{12} a_{21}\right)\left(b_{11} b_{22}-b_{12} b_{21}\right), \\
K_{5} & =\left(a_{11} a_{22}-a_{12} a_{21}\right)\left(B_{11} b_{22}-B_{12} b_{21}-B_{21} b_{12}+B_{22} b_{11}\right), \\
K_{7} & =\left(A_{11} A_{22}-A_{12} A_{21}\right)\left(B_{11} b_{22}-B_{12} b_{21}-B_{21} b_{12}+B_{22} b_{11}\right),
\end{aligned}
$$

where

$$
\begin{aligned}
& A_{11}=a_{11}^{\prime}-a_{11} c_{11}-a_{21} c_{12}+a_{11} d_{11}+a_{12} d_{21}, \\
& A_{12}=a_{12}^{\prime}-a_{12} c_{11}-a_{22} c_{12}+a_{11} d_{12}+a_{12} d_{22}, \\
& A_{21}=a_{21}^{\prime}-a_{11} c_{21}-a_{21} c_{22}+a_{21} d_{11}+a_{22} d_{21}, \\
& A_{22}=a_{22}^{\prime}-a_{12} c_{21}-a_{22} c_{22}+a_{21} d_{12}+a_{22} d_{22},
\end{aligned}
$$

and the formulas for $B_{i j}$ are obtained from those for $A_{i j}$ by interchanging $a_{i j}$ and $b_{i j}, c_{i j}$ and $d_{i j}$, while the formulas for $\mathfrak{A}_{i j}$ and $\mathfrak{B}_{i j}$ are obtained from those for $A_{i j}$ and $B_{i j}$ respectively by replacing therein $a_{i j}$ by $A_{i j}$, and $b_{i j}$ by $B_{i j}$. Every seminvariant is a function of these and their derivatives.

$A$ complete system of invariants consists of the eight invariants $I_{2}, I_{4}, \theta_{3}$, $\theta_{6}, \theta_{6}, \theta_{10}, \phi_{10}, \theta_{8}$, of which the last six are defined by the following formulas:

$$
\begin{aligned}
\theta_{3}= & I_{2}^{\prime}-2 K_{3}, \theta_{5}=I_{4}^{\prime}-2 K_{5}, \theta_{6}=4 I_{2} J_{4}-I_{2}^{\prime 2}, \\
\theta_{10}= & 3 K_{5}{ }^{2}-2 K_{5} I_{4}^{\prime}+4 I_{4}^{2} K_{7} / K_{5}, \\
\phi_{10}= & 4 I_{4} K_{6} J_{8} / K_{7}-K_{5}{ }^{2}, \\
\theta_{8}= & I_{2}^{\prime \prime}\left(I_{2}^{\prime \prime}-4 J_{4}\right)-4 I_{2} L_{6}-2 J_{4}{ }^{2}-3 I_{2}^{\prime}{ }^{2} I_{2}^{\prime \prime} / I_{2} \\
& +6 I_{2}^{\prime} J_{4}^{\prime}+15 I_{2}^{\prime}{ }^{4} / 8 I_{2}{ }^{2} .
\end{aligned}
$$

All of the invariants can be calculated by application of the jacobian process* to these eight fundamental invariants.

By means of $\left(T_{3.3}\right)$ it is possible to reduce system $\left(E_{3}\right)$ to the canonical form

$$
\begin{array}{ll}
y^{\prime}=c_{12} z+a_{11} \rho, & \rho^{\prime}=b_{11} y+d_{12} \sigma, \\
z^{\prime}=c_{21} y+a_{22} \rho, & \sigma^{\prime}=b_{22} z+d_{21} \rho .
\end{array}
$$

* Wilczynski, Projective Differential Geometry of Curves and Ruled Surfaces, p. 23. 
For this canonical form the tetrahedron $y z \rho \sigma$ is such that the two faces through $l_{y z}$ are tangent to $R_{y z}$ at $P_{y}$ and $P_{z}$, while the two faces through $l_{\rho \sigma}$ are tangent to $R_{\rho \sigma}$ at $P_{\rho}$ and $P_{\sigma}$. The lines $l_{y \rho}$ and $l_{z \sigma}$ are the common tangents of $R_{y z}$ and $R_{\rho \sigma}$. The two derivative points $z^{\prime}$ and $\rho^{\prime}$ are on $l_{y \sigma}$, while the points $y^{\prime}$ and $\sigma^{\prime}$ are on $l_{z \rho}$. The anharmonic ratios $\left(y \sigma z^{\prime} \rho^{\prime}\right)$ and $\left(z \rho y^{\prime} \sigma^{\prime}\right)$ are respectively equal to $a_{22} b_{11} / d_{12} c_{21}$ and $a_{11} b_{22} / c_{12} d_{21}$. A complete system of invariants for this canonical form consists of the eight invariants

$$
\begin{aligned}
& a_{11} b_{11}, \quad a_{22} b_{22}, \quad c_{12} c_{21}, d_{12} d_{21}, a_{11} d_{12} c_{21} / a_{22}, \\
& a_{11}^{\prime} b_{11}-a_{11} b_{11}^{\prime}, a_{22}^{\prime} b_{22}-a_{22} b_{22}^{\prime}, c_{12}^{\prime} c_{21}-c_{12} c_{21}^{\prime} .
\end{aligned}
$$

It is not difficult to show that if these invariants are given as functions of $t$, they determine a pair of ruled surfaces with generators in correspondence, except for a projective transformation.

A ruled surface and a derivative* ruled surface are a pair of ruled surfaces with their generators in correspondence. The derivative of $R_{y z}$ is generated by $l_{\rho \sigma}$, where

$$
\rho=2 y^{\prime}+p_{11} y+p_{12} z, \quad \sigma=2 z^{\prime}+p_{21} y+p_{22} z,
$$

the coefficients $p_{i j}$ being those of Wilczynski's system $(A)$. It is easy to show that in this case the coefficients of system $\left(E_{3}\right)$ are given by the formulas

$$
c_{i j}=d_{i j}=-\frac{1}{2} p_{i i}, \quad b_{i j}=\frac{1}{2} u_{i j}, \quad a_{i j}=\frac{1}{2}, a_{12}=a_{21}=0 \quad(i, j=1,2),
$$

wherein the functions $u_{i j}$ are those usually denoted $\dagger$ by these symbols. If for $R_{\rho \sigma}$ we choose the principal derivative ruled surface $\ddagger$ of $R_{y z}$, then our theory of a pair of ruled surfaces becomes a theory of the single ruled surface $R_{y z}$, because the principal derivative of a ruled surface is covariant to the surface.

\section{Partial DIFFERENTIAL EQUATIONS}

If the coördinates $x_{i}{ }^{(j)}(i, j=1, \cdots, n+1)$ of $n+1$ points $P_{i}$ are functions of $k$ independent variables $u^{1}, \cdots, u^{k}(k<n)$, then the locus of each point is a variety $V_{k}$, and we thus obtain $n+1$ such varieties in $S_{n}$ with their points in correspondence. It is possible to determine the coefficients of a system of equations of the form

$$
\frac{\partial x_{i}}{\partial u^{p}}=\sum_{j=1}^{n+1} a_{i j p} x_{i} \quad(i=1, \cdots, n+1 ; p=1, \cdots, k),
$$

* Wilczynski, loc. cit., p. 146.

† Wilczynski, loc. cit., p. 96.

† Wilczynski, loc. cit., p. 216. Stouffer, Some canonical forms and associated expansions in projective differential geometry, Address before the Southwestern Section, St. Louis, Nov. 26, 1927. 
so that $\left(x_{1}{ }^{(j)}, \cdots, x_{n+1}^{(j)}\right)(j=1, \cdots, n+1)$ will be $n+1$ sets of solutions. These coefficients satisfy $\frac{1}{2} k(k-1)(n+1)^{2}$ integrability conditions which can be obtained by demanding that the derivatives of the second order calculated by differentiating equations (21) shall be unique. These integrability conditions are

$$
\begin{aligned}
\frac{\partial a_{i j p}}{\partial u^{q}}+ & \sum_{l=1}^{n+1} a_{i l p} a_{l j q}=\frac{\partial a_{i j q}}{\partial u^{p}}+\sum_{l=1}^{n+1} a_{i l q} a_{l j p} \\
& (i, j=1, \cdots, n+1 ; p, q=1, \cdots, k ; p<q) .
\end{aligned}
$$

From these conditions, it is easy to deduce the equations

$$
\sum_{i=1}^{n+1}\left(\frac{\partial a_{i i p}}{\partial u^{q}}-\frac{\partial a_{i i q}}{\partial u^{p}}\right)=0 \quad(p, q=1, \cdots, k ; p<q) .
$$

A transformation

$$
x_{i}=\lambda_{i} \bar{x}_{i} \quad(i=1, \cdots, n+1),
$$

with the $\lambda_{i}$ supposed to be functions of $u^{1}, \cdots, u^{k}$, and a transformation

$$
\bar{u}^{p}=\bar{u}^{p}\left(u^{1}, \cdots, u^{k}\right) \quad(p=1, \cdots, k),
$$

leave each of the varieties $V_{k}$ invariant. Therefore the projective differential geometry of a set of $n+1$ varieties in $S_{n}$, with their points in correspondence, can be studied by means of the invariants and covariants of system (22) under the total transformation (24), (25).

If we use a general transformation of the form (1) with coefficients that are functions of $u^{1}, \cdots, u^{k}$, we obtain the geometry of a configuration which consists of a certain number of varieties, each of which is the locus of $\infty^{k}$ linear spaces in correspondence. For example, if $n=3$ and $k=2$, and if $\left(T_{3.1}\right)$ is used we have the geometry of a quadruple of surfaces* in ordinary space, with their points in correspondence. If $n=3$ and $k=2$, but if $\left(T_{3.8}\right)$ is used, the configuration is a pair of congruences $\dagger$ in ordinary space with their generators in correspondence.

* D. Sun, a student at Chicago, has elaborated the details of this geometry in a work as yet unpublished. This was his doctoral dissertation, August 31, 1928.

† A. J. Cook, a student at Chicago, is studying this configuration in his doctoral dissertation.

University of Chicago,

Chicago, Ill. 